Journal Of Al Azhar University Ngineering Sector

Vol. 13, No. 49, October, 2018, 1332-1344

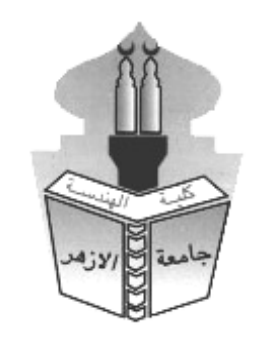

\title{
ONLINE SIGNATURE RECOGNITION AND VERIFICATION USING (SURF) ALGORITHM WITH SVM KERNELS
}

\begin{abstract}
Ali Khaleel Ibrahim Hamadly, Hossam Eldin Hassan Abdel Munim and Hoda Korashy Mohamed,

Computer and Systems Engineering, Department Ain Shams University

ABSTRACT

One important way to identify the signer is the personal signature. The operation of recognize the signature and identify it, is very important. This process may be done either offline or online.

This paper explains the online technique. Features extraction, pattern matching and Images processing are techniques used for signature confirmation. Speed up robust features (SURF) is an algorithm that uses the image of local feature with ability for matching images. SURF recognizes, describes and extracts the local feature of forged signature from the image.

SURF algorithm provides fast and accurate comparison tool that can work under different lights, visions and rotation situations to check if the person signature is original or forged. The features extracted from the SURF algorithm are entered into Bag-of-word features. The features of bagof-word are used inside multiclass Support Vector Machine (SVM) classifies. SURF with SVM kernels gives an accuracy of $98.75 \%$.

Keywords: SURF, SVM, Feature extraction

1. Introduction

Online signature check is used in different areas especially in financial sectors and banks. The signed person will sign in special pad or tablet (or any smart device), using a pen for special purpose or by his own finger. Many useful features have utilized to check the originality of the signature like (speed, writing match, pressure, etc) which will lead to increase the security and the accuracy rate [1].

The signature verification algorithms can be partitioned into two classifications: worldwide feature algorithms and local feature algorithms. Local feature extraction is steadier than global feature. They have been connected effectively in some certifiable applications, for example, protest acknowledgment, surface acknowledgment, picture recovery, robot restriction, video information mining, building scenes, and object category recognition [2].

Related works will be described in the second section. The needed definitions and principles will be described in section 3. The details of the proposed system will be described in section 4 . Process in the proposed system will be described in section 5 . Results with analysis will be discussed in section 6, while conclusion will be presented in section 7.
\end{abstract}

\section{Related works}

Berrin Yanikoglu et al [1] proposed a system used for online signature verification which based on Fourier descriptors. Preprocessing step is included by using multi sub-step before feature extraction:

1- Time normalization.

2- Stroke concatenation. 
In paper [2] by Loris Nanni et al, global features are extracted by using number of categorizer using multi-matcher for the online signature verification. The preprocessing step is profoundly reliant on hashing threshold.

Prakash [3] suggested an online mark confirmation and acknowledgment framework that is based on taking the signatures symbolic representation, by investigate the global features. Additionally they have given the idea of compose subordinate limit and highlight subordinate edge prompting beat other worldwide element -based systems.

Alisher Kholmatov et al. [4] utilized three nearby highlights. For example, organizes in respect to the main purpose of mark direction, the $\mathrm{x}$ and $\mathrm{y}$ arrange contrasts between two back to back focuses, and the ebb and flow contrasts between two successive focuses.

In [5] Harpreet Kaur proposed an offline method for signature acknowledgment by utilizing surf highlight extraction and neural systems.

\section{Definitions and Principles}

\subsection{Surf algorithm}

SURF calculation is a nearby element and descriptor calculation that can be utilized as a part of numerous applications, for example, question acknowledgment. SURF utilizes considerably in a bigger number of highlights descriptor from starting point picture which can listen to commitment of the mistakes caused by nearby variety in the normal of all the coordinating elements.

SURF can effectively realize and recognize declare in starting point pictures even if there is arise an appearance of untidiness and obstacles on the grounds that SURF has include descriptor which is invariant to scale [6]. The procedures of SURF calculation can be separated into three primary steps:

1- First step is "Identification venture": interest points' are selected at distinctive locations in the image, such as corners, blobs, and T-junctions. The most interested points are repeatability that expresses the reliability of the detector to find the same physical points.

2- Second step is "descriptive step": the main points should have unique identifier that doesn't depend on the features scale and rotation which are called descriptor. The information of interest points have represented by the descriptor.

3- Third step is "Coordinating step": in this procedure descriptor vectors are analyzed between the question picture and the new info or beginning picture, the coordinating score is computed in view of the separation between vectors e.g. Euclidian separation and the indication of Laplacian.

\subsection{Bag of words}

The bag-of-words (BOW) model is a simplifying representation used in natural language processing and information retrieval (IR). Also it is known as the vector space model [7]. In this model, a text (such as a sentence or a document) is represented as the bag of its words, disregarding grammar and even word order but keeping multiplicity. The bag of-words display has too been used for computing vision [8]. The bag of-words demonstrate is usually utilized as a part of strategies of document classification where the (recurrence of) event of each word is utilized as a feature for training a classifier [7]. In the wake of changing the content into a "bag of words", we can compute different measures to portray the content. The most widely recognized kind of qualities, or highlights figured from the Bag-of-words demonstrate is term recurrence. Image classification is a process that classifies images based on information obtained from the images [9].

The method that is using here is the (Bag-of-words) model of image classification, often known as Bag-of-feature (BOF) as well. It basically extracts the main feature of the learning class 
images and forms them into a codebook that will be used to be compared with the features of unknown classes' images to find the best class to represent these unknown classes' images [10].

For each image (during training or testing) we substitute every SURF descriptor with the nearest centroid. We calculate the number of these descriptors for each picture of every one of the centroids. For each test image we figure its pack of key focuses, feed it to the classifier and get the classification.

\subsection{Support vector machine}

Support vector machines (SVMs) are a mechanism for binary classification. SVMs can be viewed as an expansion of the perceptron, which tries to discover a hyperplane that isolates the information. The perceptron basically tries to discover any isolating hyperplane, without considering how plainly the multi-line isolates the information.

However, naturally, a hyperplane that is as far away as could be allowed from either class is best, since we anticipate this to generalize better to concealed information (an illustration is appeared in Figure (1). A specialized measure of how unmistakably multi-line a isolates information is its edge. This is the distance of the hyperplane to the closest point in the dataset; a huge edge implies that the hyperplane unmistakably separates the data.

Giyen the meaning of edge, we can characterize the objective of an SVM: for training set $(x i, y i)\}_{i}^{n}=1$, where $x i$ is the observed data and yi the labels, an SVM find a maximum margin separating hyperplane [11].

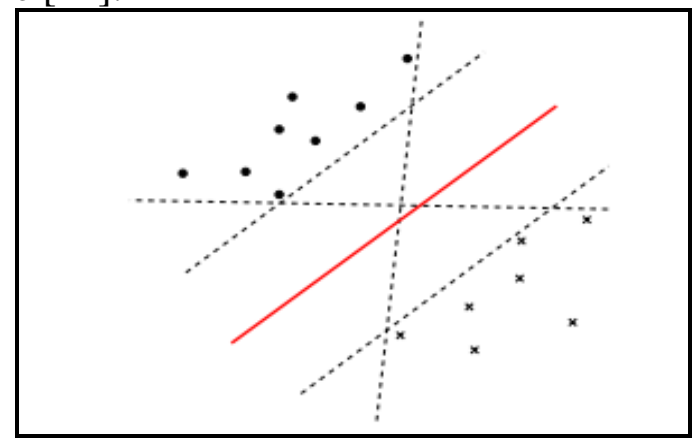

Figure 1: Multiple hyperplane disconnect dataset [11]

\section{Proposed system}

The input into a signature identification system is typically an image.

The result is a recognition or verification of an object or a number of objects appearing in the input. The proposed framework comprises of following stages.

\subsection{Input image}

In the wake of checking the mark pictures and before highlight extraction step we require preprocessing. The purpose of preprocessing is to change over colored image in to gray scale image.

\subsection{RGB to gray conversion}

In display innovation, all picture catching and filtering tools utilize shading. Subsequently, we additionally utilized a shading filtering devices to examine signature pictures. A shading picture comprises of an organize lattice and three shading networks.

Coordinate matrix contains $\left(x^{i} y\right)$ organize estimations of the picture. The shading networks are marked as red (R), green (G), and blue (B). Systems exhibited in this investigation depend on dark scale pictures, and along these lines, examined or caught shading pictures are at first changed over to dim scale utilizing the accompanying

Gray color $=0.299 \times$ Red $+0.5876 \times$ Green $+0.114 \times$ Blue 


\subsection{Proposed system implementation}

The objective is to build a system of signature recognition and practicable verification using known algorithms that are specific to the proposed system. The proposed system is implemented by using three algorithms SVM, SURF, and BOW.

\subsubsection{Methodology}

The methodology of the proposed system can be subdivided into two stages; Training operations are the processes that done inside the core of the system which it concern basically functions of training only.

Signature recognition related to the operations that performed in the overall the system where the signature image to be verified is entered and obtain the result that will be filled out with full details and information about the signature holder.

\section{1-First Dataset (universal English)}

The international dataset has chosen from German website contain ten person signatures. Every person has twenty-four sign, so the total signs that entered to the system two hundred forty signs subdivided into two subs. Eighty percent for training and twenty percent for the test. The number of signs for training about one hundred sixty and it will pass through series of algorithms until it classified into ten classes, he remaining signs will be eighty sings will be entered for the test as shown figure 2.

\section{2-Second Dataset (Arabic)}

The second dataset has (768) signature divided into five hundred and twelve for training and two hundred and fifty -six for the purpose of testing, as well Part of it is built using three different printers with different levels of noises. The first has few noise and the second has medium noise and the third has high noise as shown in the figures 3,4 and 5.

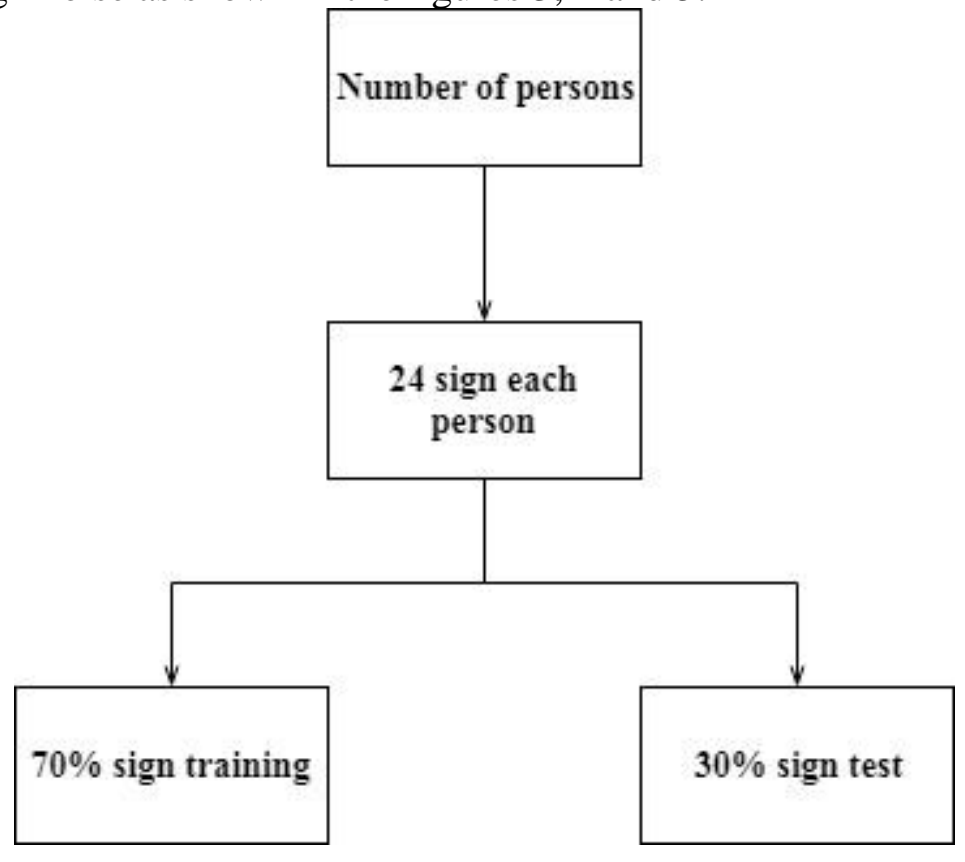

Figure 2: Dataset 1 


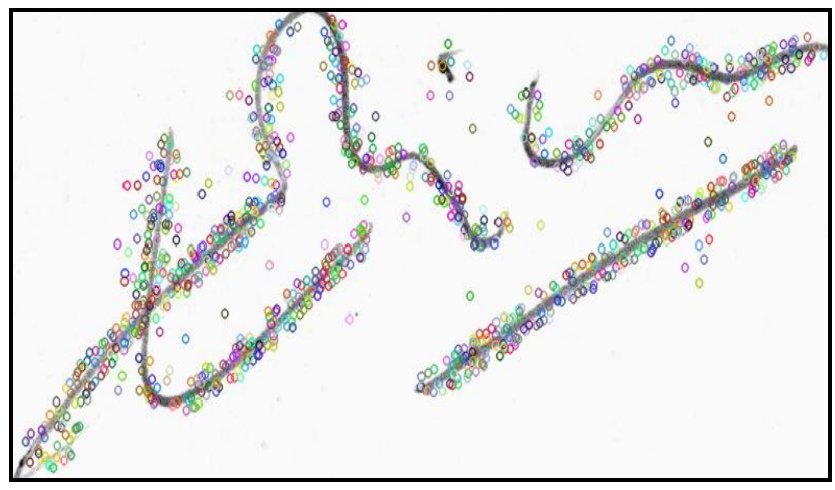

Figure 3: Dataset with low noise

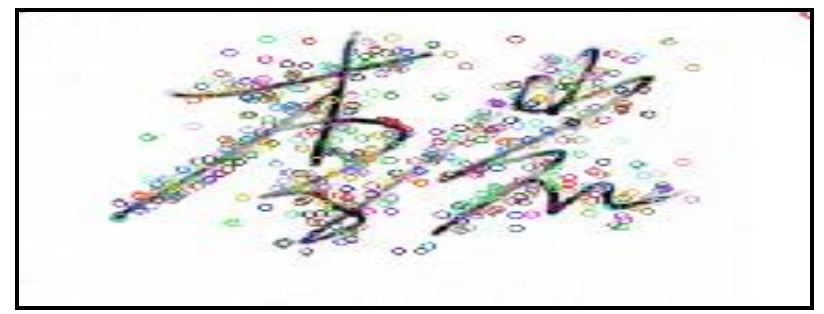

Figure 4: Dataset with medium noise

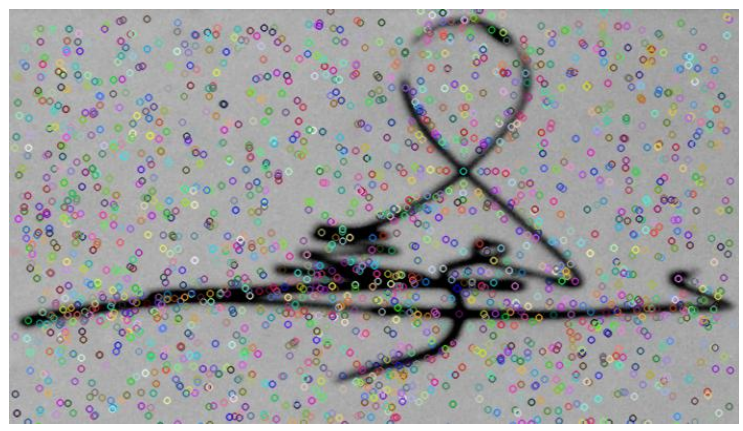

Figure 5: Dataset with high noise

\subsection{SURF algorithm Implementation}

SURF algorithm will be constructed for features extractions:

- Key point: points that indicate feature existence.

- Descriptor: translator that need (lighting, angle, rotation, edge, so on). Its job that entering one hundred sixty signs to SURF algorithm and through it determine to each sign large group key point and descriptor for exclude features preparing it for second stage. The urgent reasons to use SURF algorithm are:

- Take the sign in any form

- No need to resize

- No need for all operations before reprocessing that leads to accelerate system.

\subsection{Bag of word (BOW) Implementation}

This algorithm works as a dictionary to extracted vocabulary like numbers or words that exist in descriptor which are not repeated. It means that use the strongest features for system entering with ignoring features have low repetitions because it is not important in front of powerful features or repetitions. The BOW algorithm depends on two methods:

1. K-Means

2. $\mathrm{F}-\mathrm{KNN}$ 
The benefits of BOW are arrangement features in form of dictionary to be easy deal with it as well as display strongest features and finally accelerate system.

\subsection{Support vector machine (SVM) Implementation}

After SURF and BOW algorithm, data involvement descriptor for training to be isolate and classify into ten classes by using classifier. Every one carries specific ID until ID10.

\subsection{Prediction}

After SVM process, check and test process started by admission the left eight signs for each person which will be eighty sign and the previous operations are performed using the same algorithms (SURF, BOW). Then send to the classifier to predict for identify and match to any person or ID. When the test and train operations complete the accuracy of the system will be (98.75).

\section{Processes within the implemented system}

We have two interfaces programmed by Java programming language. First interface is for client and the second interface is for server. Steps that the system pass through it is shown in figure (6). The two interfaces will be displayed as shown in figure (7) and figure (8).

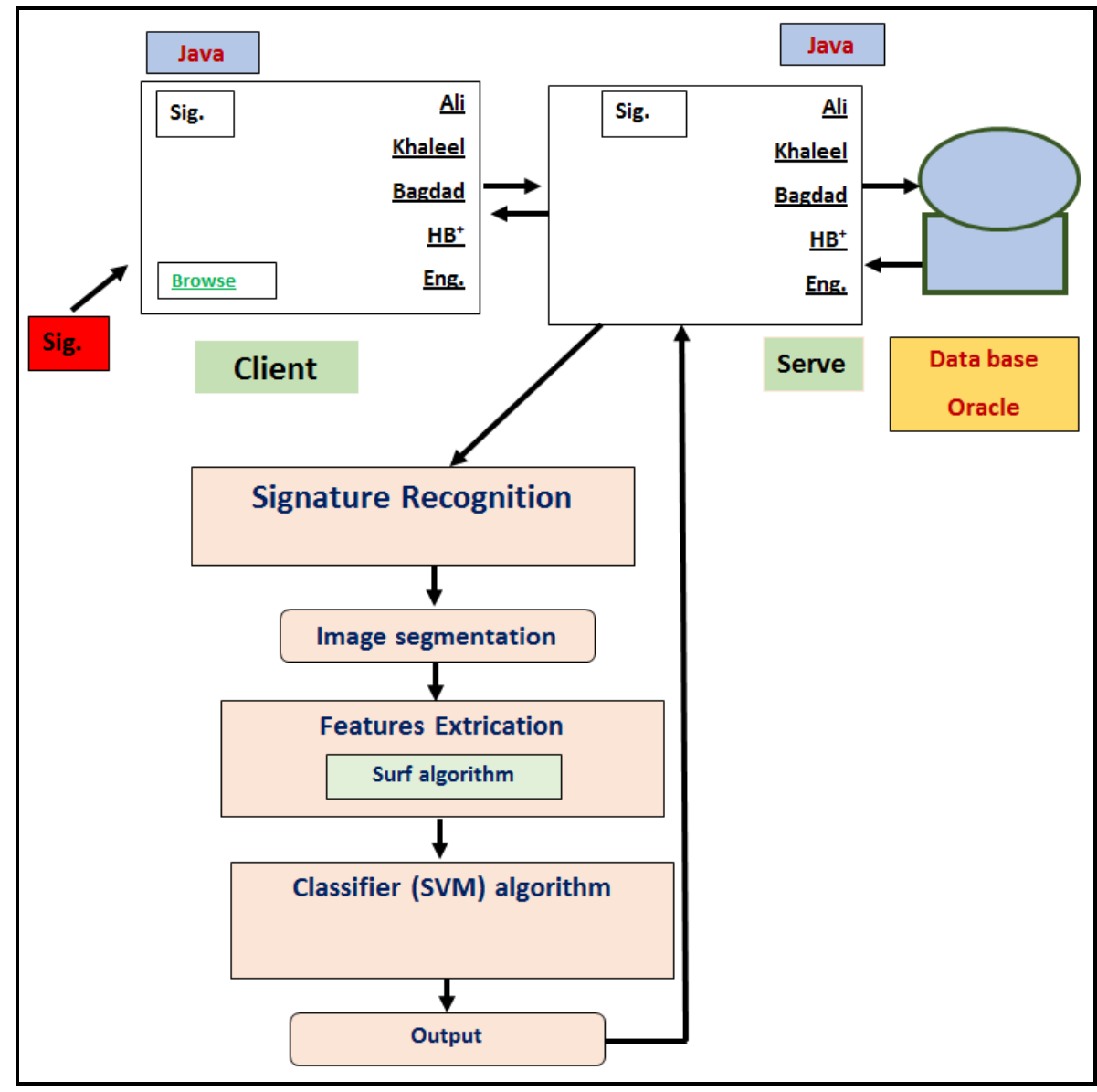

Figure 6: General structure of online signature recognition system 


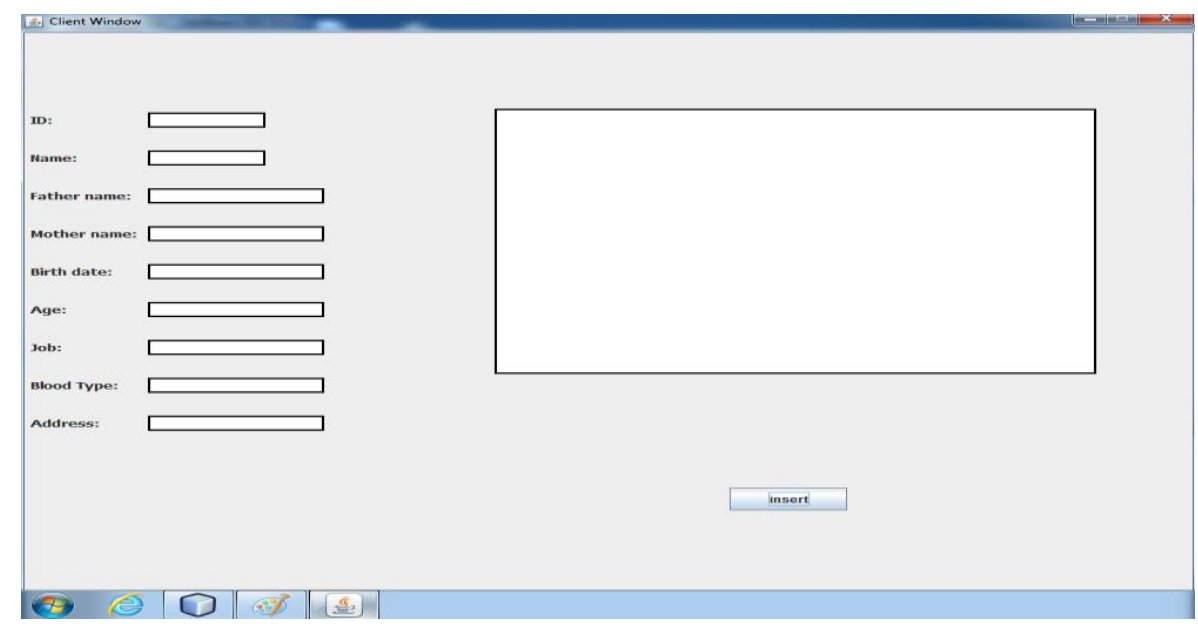

Figure 7: Client interface

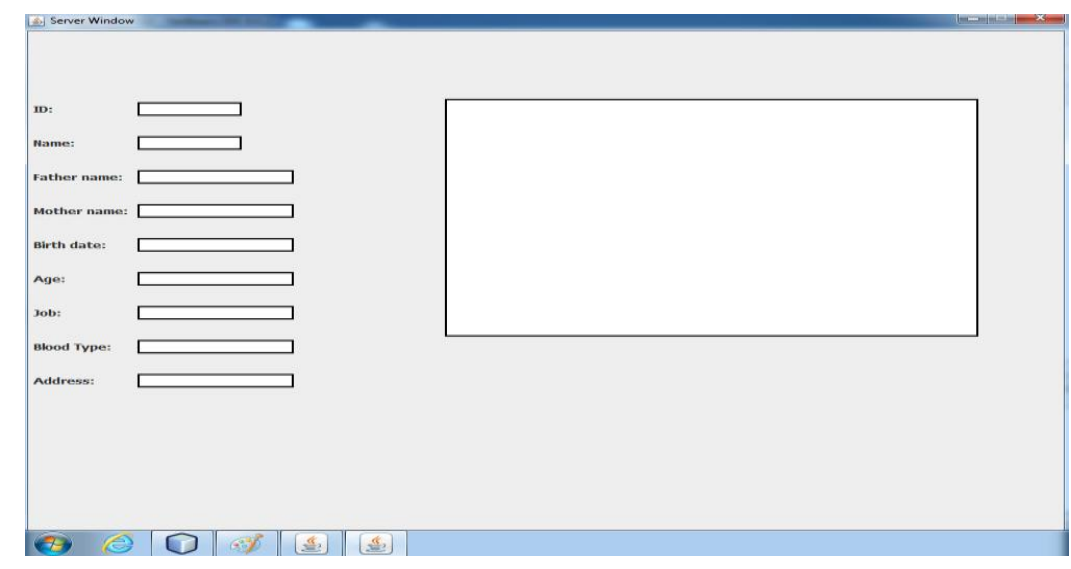

Figure 8: Server Interface

\section{Signature upload}

The signature image is uploaded after pressing browse button form program interface of the client that programmed by Java language.

The image of the signature is transferred to the second interface server as shown in the image in figure 9 with the sending of a post.

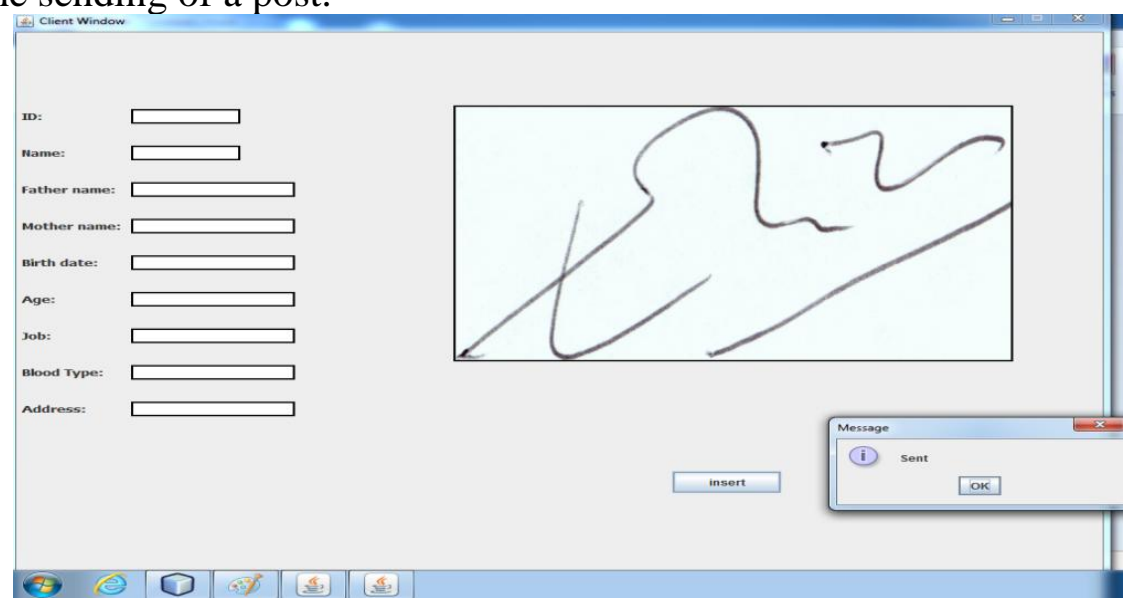

Figure 9: Download signature on the Client interface 


\section{Processing}

This stage is one of the most important stages where the process of prediction has occurred. The image is sent from the server interface to the language of programming $\mathrm{VC}++$ as shown in the general plan where the processes are returned, including surf algorithm by identifying the key point and interpreter in the extraction of features and then enter the algorithm (BOW) and identify the signature by matching the previous workbook with which we conducted the training process.

\section{Determine ID}

At this point, ID is retrieved from the prediction to the server interface again.

\section{Data base}

ID is sent from the server interface to the database where there are tables for all information about each ID such as name, father's name, mother's name, workplace, blood category ... Bring any ID to Oracle) matches the information in which it is stored to identify the person who is signed (figure 10).

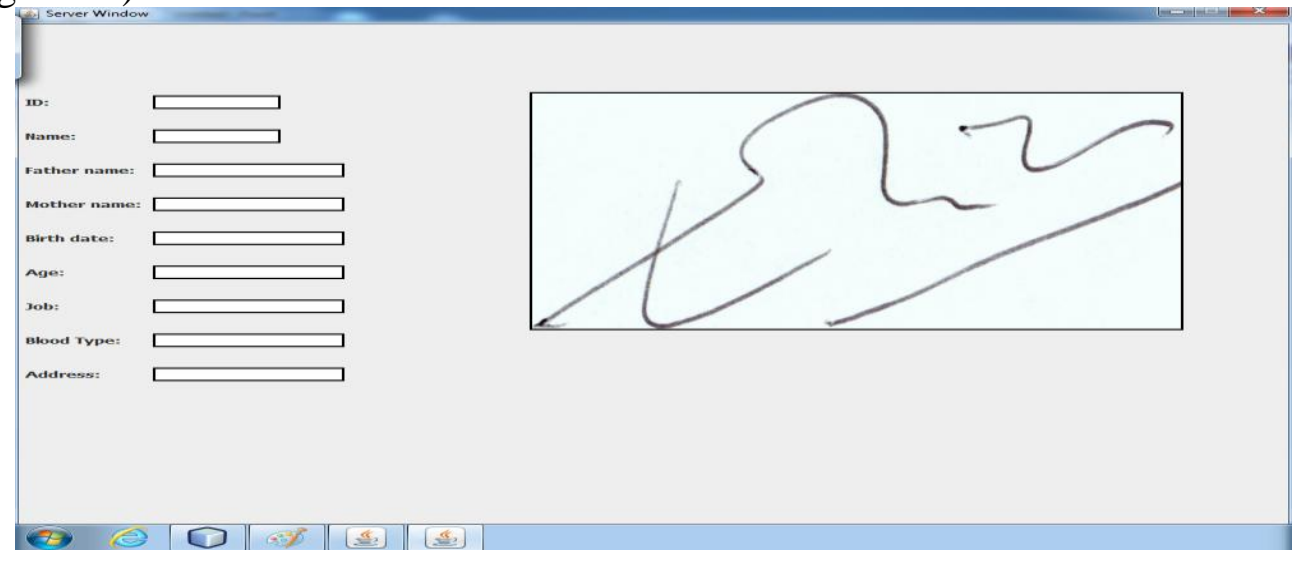

Figure 10: Send signature on the Server interface

\section{Data base(oracle) to server}

The database retrieves all information and data to the server interface in the form of a table that contains all the details of the ID, figure 11.

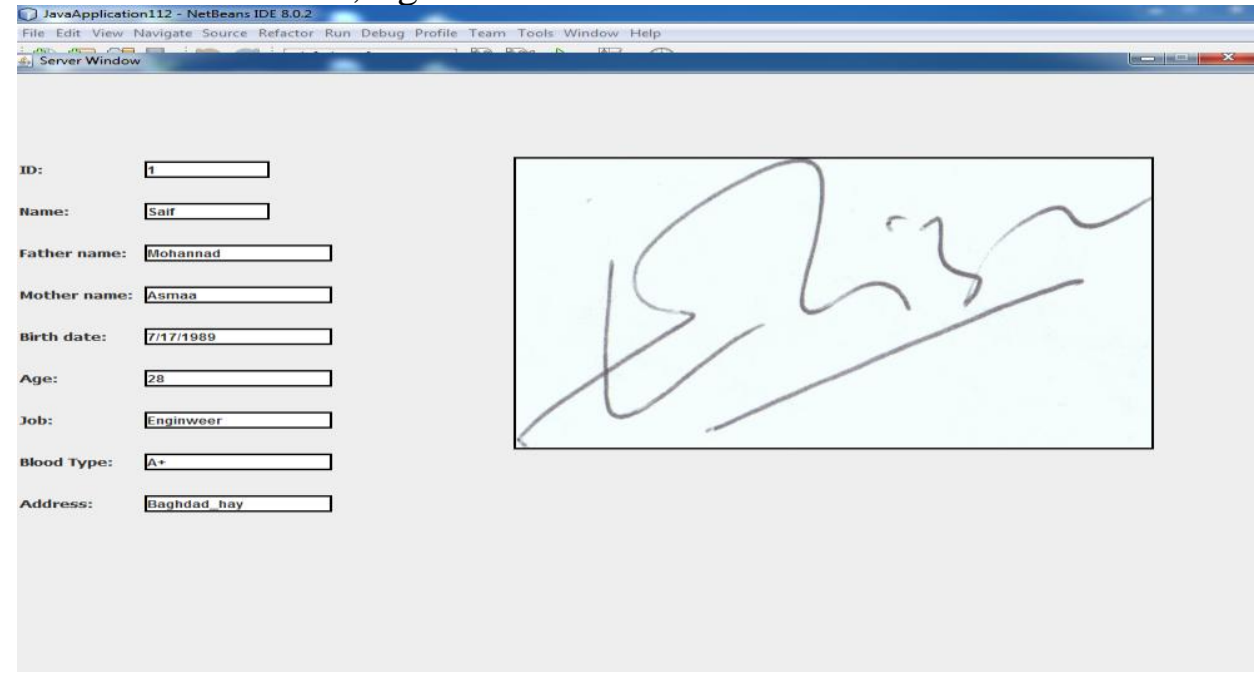

Figure 11: Receive information on server interface 


\section{Signature recognition}

It is the last stage that all information and data are transferred to the client interface, which appears as a table showing all the required information about the identity of the sign holder as shown in figure 12.

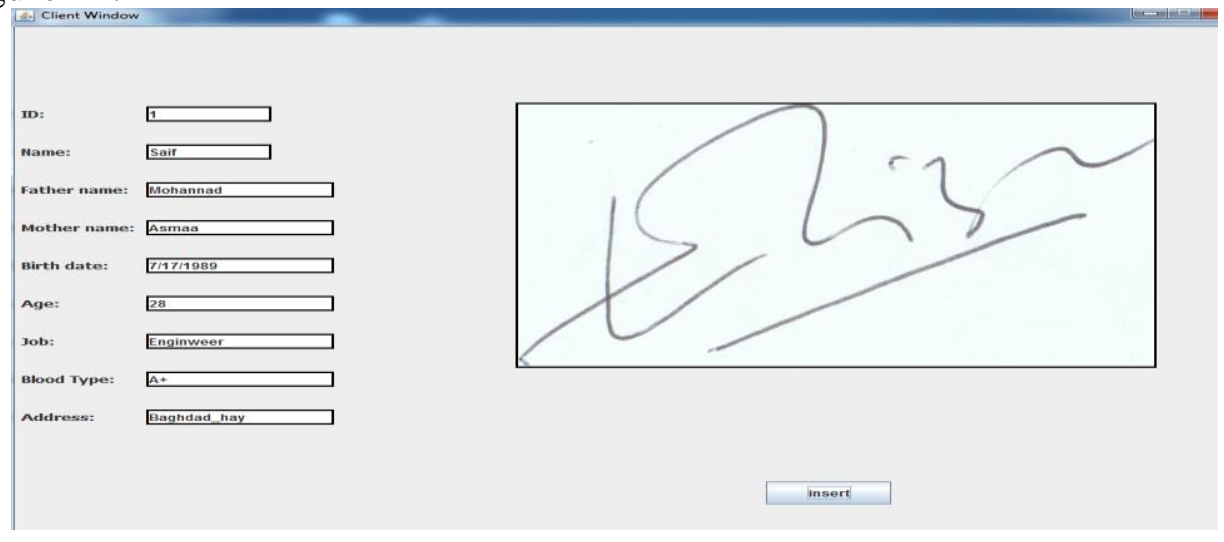

Figure 12: Receive information on client interface

\section{Results and analysis}

\subsection{Experiment 1}

Compare the system resolution between the different data set used in the proposed system.

The first dataset contains 240 signs that are taken from ten persons, details are found in table (1). The second dataset contains 768 signs that are taken from 32 persons details are found in table (2).

According to the results in table (1) the nonlinear radial base function (RBF) shows an accuracy of $98.75 \%$ where the linear shows $98.75 \%$ and ploy $=1$ provide full accuracy with $100 \%$ and poly $=2$ provide us with $77.5 \%$ accuracy.

According to the results in table (2) the nonlinear (RBF) shows an accuracy of $97.7 \%$ Where the linear shows $97.7 \%$ and ploy $=1$ provide accuracy $97.3 \%$ and poly $=2$ provide us with $71.1 \%$ accuracy.

Figures 13, 14, 15 and 16 will show the comparison elements.

Table 1: 10 persons $=240$ sig

\begin{tabular}{|l|l|l|}
\hline Technique & Accuracy & Comments \\
\hline nonlinear (RBF) & $98.75 \%$ & Precision=No hit/No test=79/80=98.75 \\
\hline Linear & $98.75 \%$ & Precision $=$ No hit/No test=79/80=98.75 \\
\hline Poly=1 & $100 \%$ & Precision $=$ No hit $/$ No test $=80 / 80=100$ \\
\hline Poly=2 & $77.5 \%$ & Precision $=$ No hit/No test=62/80=77.5 \\
\hline & & \\
\hline
\end{tabular}

Table 2: 32 persons $=768$ sig

\begin{tabular}{|l|l|l|}
\hline Technique & Accuracy & Comments \\
\hline nonlinear (RBF - SVM) & $97.7 \%$ & Precision=No hit/No test=250/256=97.7 \\
\hline Linear (SVM) & $97.7 \%$ & Precision $=$ No hit/No test=250/256=97.7 \\
\hline Poly=1 (SVM) & $97.3 \%$ & Precision $=$ No hit/No test=249/256=97.7 \\
\hline Poly=2 (SVM) & $71.1 \%$ & Precision $=$ No hit/No test=182/256=71.1 \\
\hline
\end{tabular}


ONLINE SIGNATURE RECOGNITION AND VERIFICATION USING (SURF) ALGORITHM WITH SVM KERNELS

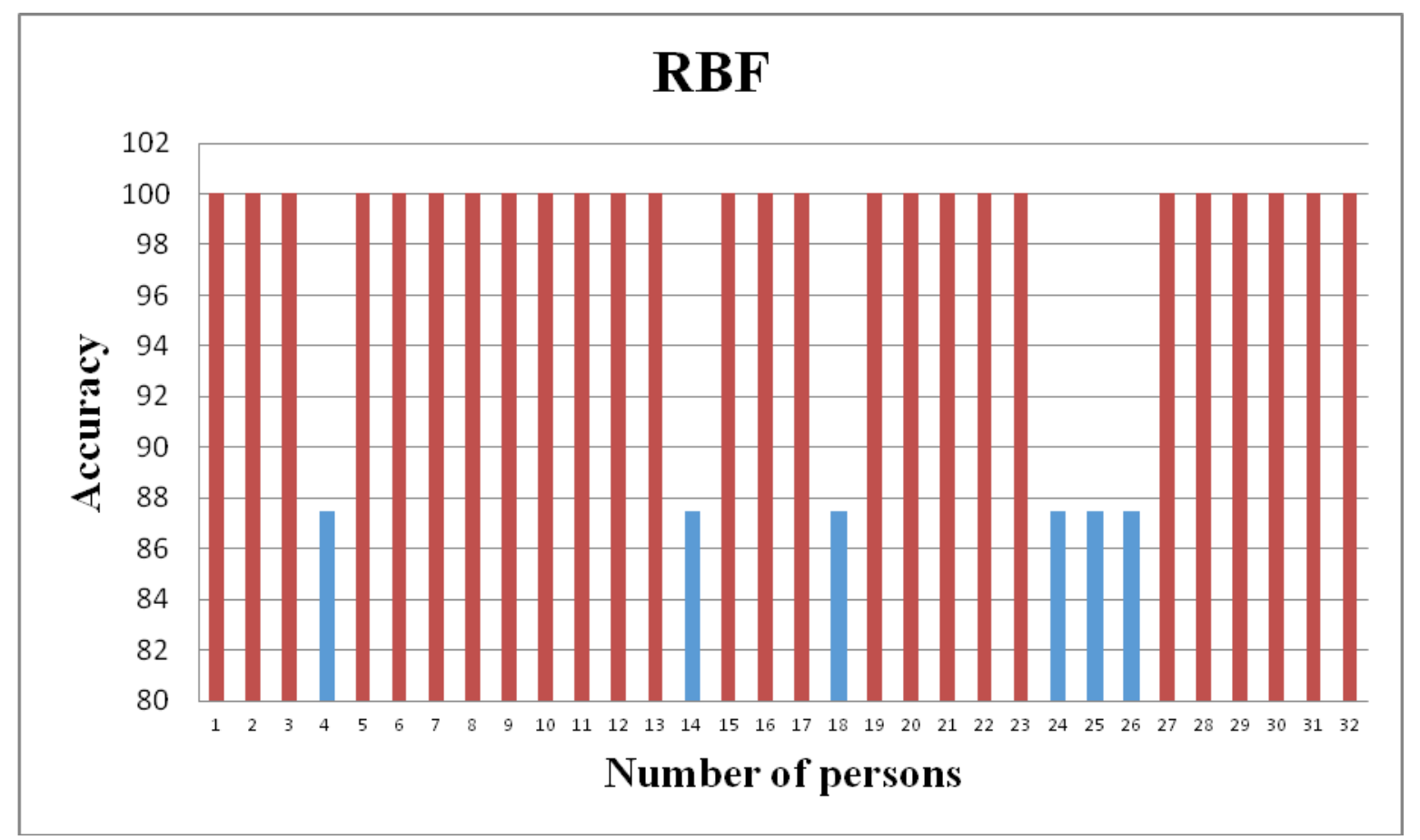

Figure 13: Non-linear RBF

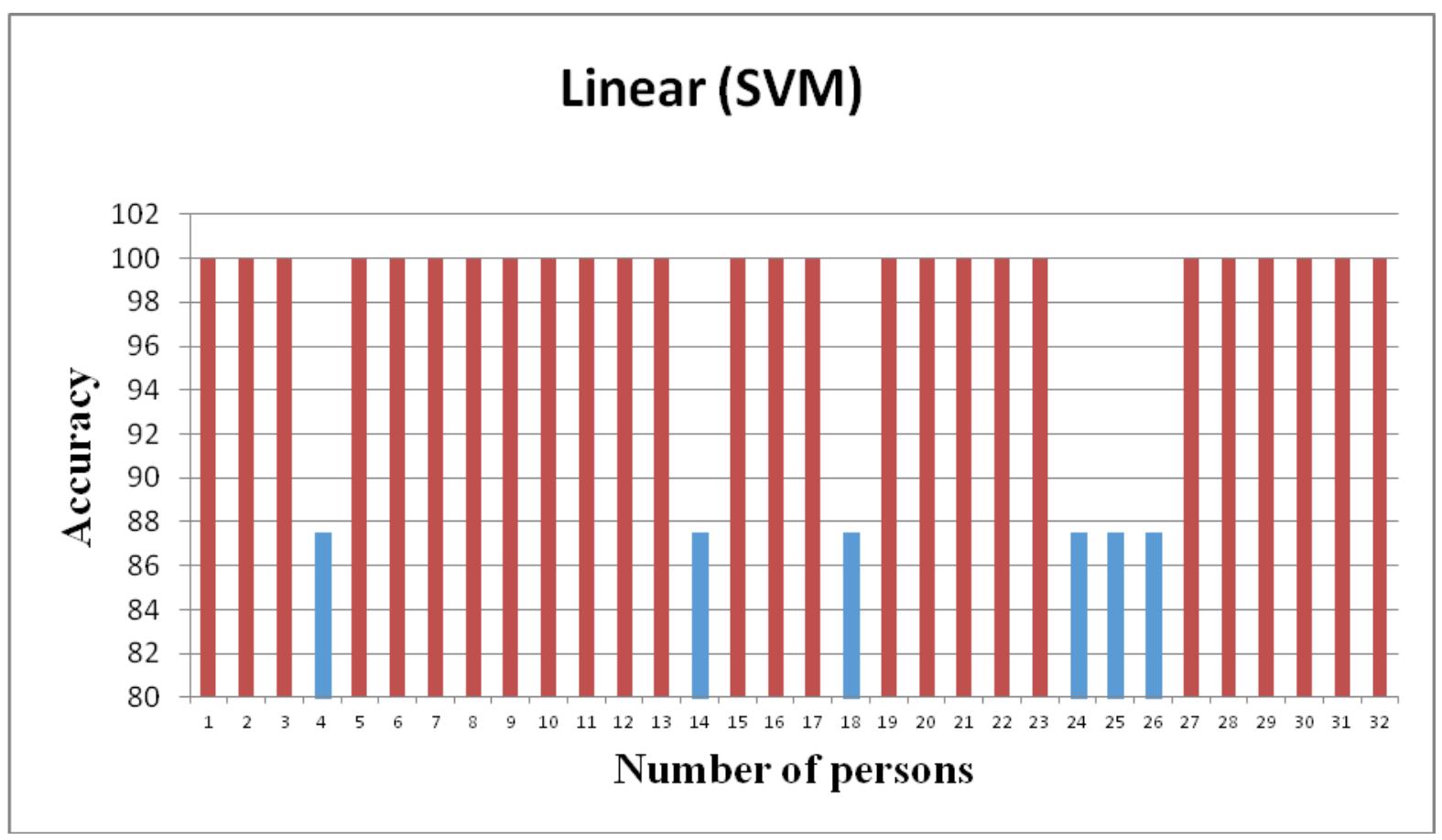

Figure 14: Linear (SVM) 


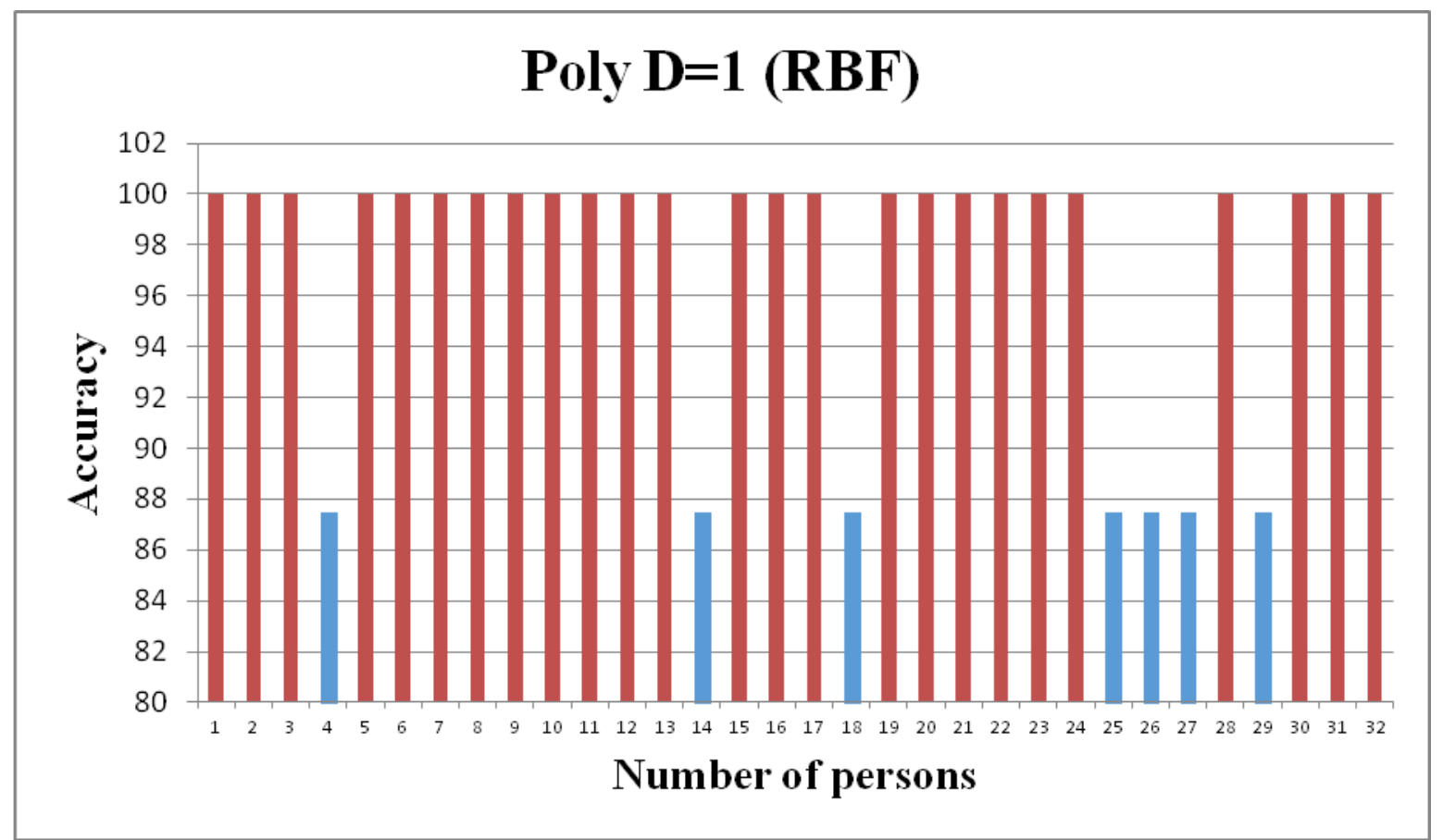

Figure 15: Non-linear Polynomial (degree 1)

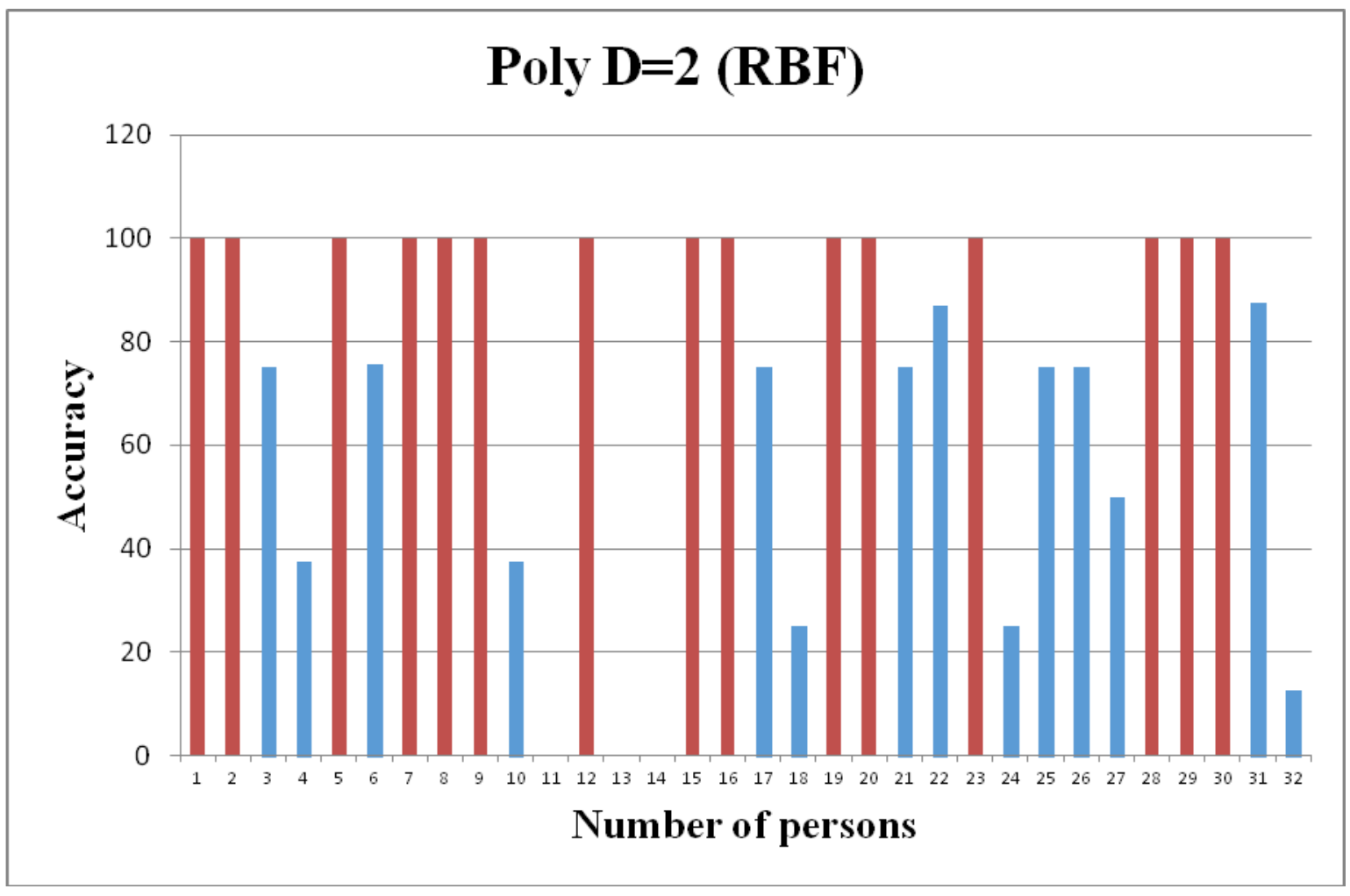

Figure 16: Non-linear Polynomial (degree 2) 


\subsection{Experiment 2}

Comparison between the proposed signature recognition system and other system; are shown in table 3.

In table (3) many related works is compared to the proposed system and the proposed results. Porwik and Para[12] used Combined method with hough transformation as classifier and they got a recognition of 94.60. Pansare and Bhatia[13] used Back propagation neural network (NN) as classifier and they got a recognition of 82.60. Ubul et al [14] used Euclidean distance (ED) as classifier and they got a recognition of 86.45.

Other researchers use other classifiers such as K-nearest neighbor $(\mathrm{K}-\mathrm{NN})$ which provide a rate of 93.53; Bayes technique with rate 89.26 and SURF with rate 90.

The recognition phase in our proposed system use different techniques with rates as follow: SURF (SVM-RBF) with recognition 98.75, SURF (linear) with recognition 98.75, and Surf (polynomial $\mathrm{D}=1$ ) with recognition 100

Experiment results show improvement in the recognition phase.

Table 3: Comparison between proposed system and other systems

\begin{tabular}{|l|l|l|l|}
\hline & Author \& reference & \multicolumn{1}{|c|}{ Classifier } & $\begin{array}{l}\text { Recognition } \\
\text { Rate (\%) }\end{array}$ \\
\hline 1 & Porwik and para [12] & Combined method with hough transformation & 94.60 \\
\hline 2 & Pansare and Bhatia [13] & Back propagation NN & 82.60 \\
\hline 3 & Ubul et. Al .[14] & Euclidean distance (ED) & 86.45 \\
\hline 4 & & K-nearest neighbor (K-NN) & 93.53 \\
\hline 5 & & Bayes & 89.26 \\
\hline & & SURF & 90 \\
\hline & The proposed system & SURF (SVM-RBF) & 98.75 \\
\hline & The proposed system & SURF (linear SVM) & 98.75 \\
\hline & The proposed system & SURF (polynomial D=1) & 100 \\
\hline
\end{tabular}

\section{Conclusion}

In this study we presented Online Signature Recognition and Verification using SURF with SVM Kernels

The principles that were applied in general were as follows:

First: the principle of extracting the image processing feature using algorithms (SURF / BOW [K-mean, F-KNN])

Second: the principle of course: using SVM with kernels (linear and non-linear [RBF, polynomial "degree 1 and 2"

Third: the principle of the database as the source of information using Oracle

Fourth: the principle of transmission and receive as a network using the TCP / IP protocol, has been used to complete the protocol to deliver data without any shortage.

Two data sets were used in this paper the first one which is found in [15] the collection contains simultaneously acquired online and offline samples, which was lunched to IEEE conference in Germany with $98.75 \%$ correct matching in short period.

Second data sets collected by authors for Arabian people and acquire to computer using digital pens, tablet and scanner

The system was tested using two types of data set, the universal-based with 240 signatures of noise and the second large containing 768 signatures of the group and integrated with random signatures with the passage of the socket of different printers. It was found that the accuracy of the system did not influence of the increase of the data set.

Advantages of the proposed system include:

1. Do not use pre-processing processors (such as image resizing, thinning, color). 
2. Explanation: the speed of implementation in the system online and take pictures to know and receive the immediate result.

3. We have proofed that the proposed system has high accuracy no matter how large the data set.

Authors use Signature Verification Technique using neural network and svm with surf feature.

The results of matching are improved as we use neural network and svm with surf feature technique for matching. Better improved quality of signature and matching results are obtained.

\section{REFERENCES}

[1] Sae-Bae, Napa, and Nasir Memon. "Online signature verification on mobile devices" , IEEE Transactions on Information Forensics and Security 9.6 (2014): 933-947.

[2] Abaza, Ayman, et al. "A survey on ear biometrics.", ACM computing surveys (CSUR) 45.2 (2013):

[3] Plamondon, Réjean, Giuseppe Pirlo, and Donato Impedovo. "Online signature verification." , Handbook of Document Image Processing and Recognition, Springer London, 2014. 917-947.

[4] Parodi, Marianela, Juan C. Gomez, and Marcus Liwicki. "Online signature verification based on Legendre series representation: Robustness assessment of different feature combinations." , Frontiers in Handwriting Recognition (ICFHR), 2012 International Conference on. IEEE, 2012.

[5] Kaur, Harpreet, and Simarjeet Kaur, "Disconnected Hindi Signature Recognition Using Surf Feature Extraction and Neural Networks Approach.", 2011

[6] Tawfiq A. Al-asadi, Ahmed J. Obaid," Object detection and acknowledgment by utilizing improved Speeded Up Robust Feature", IJCSNS International Journal of Computer Science and Network Security, VOL.16 No.4, April 2016.

[7] Michael McTear \& Zoraida Callejas, "The Conversational Interface", Springer International Publishing, ISBN: 978-3-319-32965-9, Switzerland 2016.

[8] Josef Sivic and Andrew Zisserman, "Efficient visual inquiry of recordings give a role as content recovery", IEEE TRANSACTIONS ON Pattern analysis and machine intelligence, ,2012 VOL. 31, NO. 4. IEEE. pp. 591-605.

[9] NM Ali, MS Karis, AFZ Abidin, B Bakri \& NRA Razif., "Activity Sign Detection and Recognition: Review and Analysis ", 2015, Journal Technology 77(2), pp. 107-113.

[10] Nursabillilah Mohd Ali and Soon Wei Jun, "Object Classification and Acknowledgment utilizing Bag-of-Words (BoW) Model", IEEE twelfth International Colloquium on Signal Processing \& its Applications, 4 - 6 March 2016, Melaka, Malaysia.

[11] Yedukondalu, B., and A. Daveedu Raju. "New approaches of Data Mining for the Internet of Things with systems: Literature Review and Compressive." (2017).

[12] Oloyede, Muhtahir O., and Gerhard P. Hancke. "Unimodal and multimodal biometric sensing systems: a review." IEEE Access 4 (2016): 7532-7555.

[13] Pansare A and Bhatia S. "Handwtitten signature verification using Nural Network , 'international journal of applied information system (IJAIS), VOL 1,PP. 44-49, 2012.

[14] Ubul K, Adler G, Abliz M. Yasheng and Hamudlla A. Off-line Yughur signature recognition based on modified grid information features", in International conference on Information Science, Signal Processing and their applications, Canada, 2012, pp. 10561061.

[15] Liwicki, Marcus, et al. "Signature verification competition for online and offline skilled forgeries (sigcomp2011)." Document Analysis and Recognition (ICDAR), 2011 International Conference on. IEEE, 2011. 